cytic leukaemia during the course of chronic lymphocytic leukaemia: correlation of blood, marrow and spleen morphology and cytogenetics. Blood 1974;43:333-8.

${ }^{5}$ Quesenberry P, Levitt L. Haemopoietic stem cells. $N$ Engl J Med 1979;301: 755-9.

\section{Methods of early detection of systemic or local vascular disease}

There is critical need for useful methods for the early clinical detection of systemic (or local) vascular disease. The rectal biopsy technique described by Tribe $e t$ al ${ }^{1}$ provides convincing objective evidence of vasculitis and parallels my experience ${ }^{2} \mathbf{3}$ in the study of nasal biopsies in systemic or local vascular diseases. The presence of vascular changes in nasal biopsies should alert the pathologist and surgeon and contribute to the early and correct diagnosis of the diseases forming the midfacial granuloma syndrome. ${ }^{4}$ Vasculitis provides the pathogenetic basis for linking under the same heading, idiopathic pleomorphic midfacial granuloma (Stewart's type) and giant cell Wegener's granuloma and granulomatosis. It is interesting to note in this context that the material examined by Tribe et $a l^{1}$ has included rheumatoid vasculitis, polyarteritis nodosa and what the authors called "overlap syndromes" indicating the terminological difficulties when describing some of these often rare diseases.

I FRIEDMANN

Department of Histopathology, Northwick Park Hospital and Clinical Research Centre, Watford Road, Harrow, Middlesex HAI 3UJ

\section{References}

1 Tribe CR, Scott DG, Bacon PA. Rectal biopsy in the diagnosis of systemic vasculitis. J Clin Pathol 1981;34:843-50.

${ }^{2}$ Friedmann I. The changing pattern of granulomas of the upper respiratory tract. J Laryngol $1971 ; 85: 631-82$.

${ }^{3}$ Friedmann I, Sando I, Balkany T. Idiopathic midfacial granuloma $J$ Laryngol 1978;92:601-11.

4 Friedmann I. Midline granuloma. Ann Intern Med 1977;86:663.

${ }^{5}$ Friedmann I, Osborn DA. The midfacial granuloma syndrome In: Pathology of granulomas and neoplasms of the nose and paranasal sinuses Chapter 10. Edinburgh: Churchill Livingstone, 1981 (in press).
Double embedding in agar/paraffin wax as an aid to orientation of mucosal biopsies

Orientation of intestinal mucosal biopsies so that histological sections can be cut perpendicularly to the epithelial surface is of paramount importance to their histological examination and reporting. ${ }^{1}$ Many biopsies of both small and large intestine yield little or no useful diagnostic information because correct orientation has not been achieved.

Orientation of strips of mucosa in the laboratory is greatly facilitated if they are allowed to adhere, flat, to a piece of frosted glass ${ }^{2}$ or thin card. ${ }^{3}$ However, although such a procedure can be easily implemented in gastroenterological units, many intestinal biopsies, particularly rectal biopsies, are taken in diverse, non-specialised clinics and often reach the laboratory irregularly curled-up in formol saline and are a significant potential cause of wasted laboratory and clinical time and effort.

To increase the precision of orientation of all biopsies, regardless of their state on reaching the laboratory, we decided to use a method of embedding the tissue in the fixed but unprocessed state using a technique based on embedding in agar before processing into paraffin wax. - $^{-6}$

The formalin-fixed biopsy fragments are embedded in molten $1 \%$ aqueous agar at $45^{\circ} \mathrm{C}$, under dissecting microscopic control. The agar solution has several remarkable useful properties; although its melting point is $98^{\circ} \mathrm{C}$, gelling does not occur until the temperature falls to $42^{\circ} \mathrm{C}$. Solidification is fairly slow and correct orientation of a biopsy, even if curled, is easily achieved by gentle manipulation with a mounted needle during cooling. Moreover, the agar is colourless and transparent in both liquid and gel forms so that the process of orientation can be viewed through a dissecting microscope. Even photography of the dissecting microscopic appearance is possible at this stage. The embedding in agar can be conveniently performed in standard moulds, such as those used for preparing paraffin wax blocks-for example, Tissue Tek.

The block of solidified agar, with its embedded biopsy is trimmed with a scalpel or razor blade so that it presents a flat surface perpendicular to the epithelial surface and it is then processed through alcohols and chloroform to paraffin wax in the usual way. The agar does not shrink appreciably during processing and is im- pregnated by wax just as if it were tissue. Paraffin sections are cut in the usual way. No technical difficulties have been experienced; the agar cuts just as if it werewax. On staining, the agar takes up a $\frac{\bar{O}}{0}$ minimal amount of eosin and can just be $\overline{\bar{c}}$. seen as a very faint background stain but $\vec{\nabla}$ does not interfere with the histological $\unrhd$ appearances. Quantification and photomicrography are unimpeded.

This technique has been used in our laboratories now for three years. Although $\vec{\overrightarrow{ }}$ it is unnecessary for well presented, $\stackrel{\sigma}{\circ}$ mounted biopsies or for tiny oesophageal and gastric biopsies it has proved in-? valuable for rendering small, unmounted, curled and apparently "unorientatable" $\omega$ biopsies reportable.

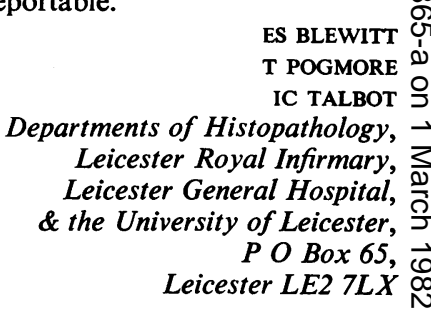

References

${ }^{1}$ Rubin CE, Brandborg L, Phelps PC, Taylor HC. Studies of celiac disease. 1: The apparent identical and specific nature of the duodenal and proximal jejunal lesion in celiac disease and idiopathic sprue. Gastroenterology 1976;38:28-49.

2 Morson BC, Dawson IMP. Gastrointestinal pathology 2nd ed. Oxford, London, Edinburgh and Melbourne: Blackwell, 1979.

${ }^{3}$ Lee FD, Toner PE. Biopsy pathology of the small intestine. London: Chapman and Hall, 1980.

4 Janisch R. Oriented embedding of single cell organisms. Stain Technol 1974;49:157-60.

${ }^{5}$ Arnolds WJA. Oriented embedding of small objects in agar-paraffin, with reference marks for serial sections. Stain Technol 1978;53:287-8.

6 Van Der Wal VP, Dohmen MR. A method for the orientation of small and delicate objects in embedding media for light and electron microscopy. Stain Technol 1978; 53:56-7.

\section{Culture of Chlamydia}

There is currently much interest in the role and importance of Chlamydia as pathogens both in adults and neonates. Isolation is still a complicated procedure and we have devised a simplified method 